Herzschr Elektrophys 2021 · 32:423-424 https://doi.org/10.1007/s00399-021-00827-7 Angenommen: 19. Oktober 2021

(c) The Author(s), under exclusive licence to Springer Medizin Verlag GmbH, ein Teil von Springer Nature 2021

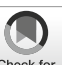

\section{Warum die Publikation von Case Reports so wichtig ist}

\author{
Carsten W. Israel \\ Klinik für Innere Medizin - Kardiologie, Diabetologie \& Nephrologie, Evangelisches Klinikum Bethel, \\ Bielefeld, Deutschland
}
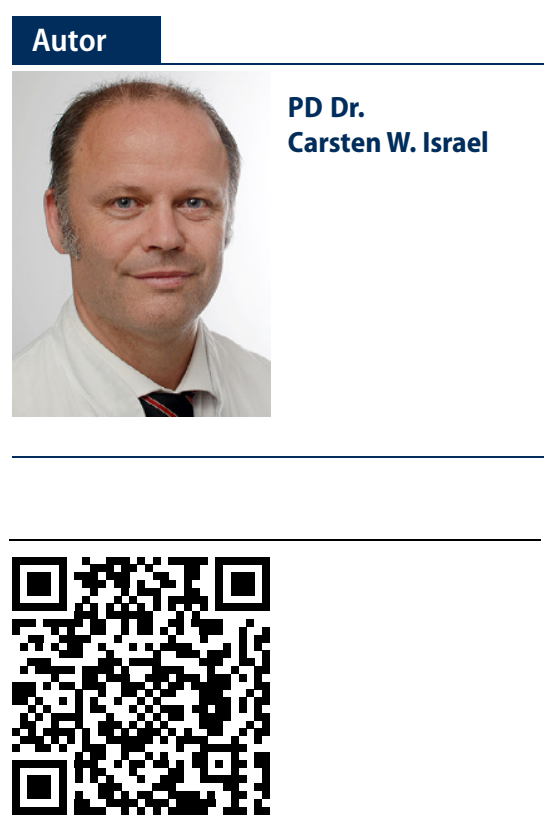

QR-Code scannen \& Beitrag online lesen
Patientenfälle, Case Reports, stellen eine Studie mit $n=1$ dar, sind also im Zeitalter der "evidence-based medicine" so ziemlich das unseriöseste und unattraktivste, was publiziert wird. Und weil Case Reports kurz sind und nie zitiert werden, machen sie den Impact Factor eines wissenschaftlichen Journals kaputt. Je weniger Zitierungen pro Jahr im Zähler und je mehr Artikel pro Jahr im Nenner, desto niedriger der Impact Factor.

Deswegen haben viele medizinische Fachzeitschriften Case Reports komplett verbannt und einen Ableger der Zeitschrift nur für Case Reports entwickelt. Der Name der Zeitschrift wird dann durch „... Case Reports" verlängert, und sie ruinieren nicht mehr den Impact Factor des Hauptheftes. Sie werden dann allerdings nicht mehr zufällig von den Lesern des Hauptheftes gelesen, aber es gibt eben Prioritäten im System wissenschaftlicher Publikationen.

Manche Zeitschriften veröffentlichen nur "abbreviated case reports" oder „images in medicine" (nur ein Bild mit Legende, sonst kein Text!), die weniger als 1 Seite lang sind. Diese werden von Thomson und Reuters nicht bei der Berechnung des Impact Factors berücksichtigt.

Ein hoher Impact Factor bedeutet einen guten Platz im Ranking der Zeitschriften eines Fachbereiches, damit hohes Prestige und mehr eingereichte hochwertige Manuskripte. Case Reports verursachen einen niedrigeren Impact Factor, damit ein schlechteres Prestige und schlechtere oder gar keine Manuskripte, ein Teufelskreis. Der Platz, der durch den Verzicht auf Case Reports frei wird, kann übrigens durch artikelbegleitende Editorials besetzt werden, die den Impact Factor der Zeitschrift durch
Zitierung des besprochenen Artikels steigern ...

Autoren müssen bei diesem System meist mitmachen, denn an vielen Universitäten werden die Forschungsgelder nach den in den Publikationen erzielten Impact Factors verteilt, und nicht nur dabei ist es sicherlich sinnvoller, z.B. 158 Punkte bei Circulation abzuholen als beispielsweise 1,5 Punkte für eine Publikation im J Interv Card Electrophys. In Letzterem müsste man etwas mehr als 100 Publikationen veröffentlichen, um so viele Forschungsgelder wie mit einem Circulation-Paper zu erwerben ...

Ist denn dann ein Heft voller Case Reports nicht regelrechter Selbstmord für Herzschrittmachertherapie und Elektrophysiologie $(H+E)$ ? Glücklicherweise nicht, denn wir können uns eine andere Prioritätensetzung leisten. Bei $H+E$ haben die Themen und Formate, die sich die Leserschaft wünscht, eine höhere Priorität als der Impact Factor, und unsere Leserschaft hat sich in der letzten Umfrage unter anderem eine besonders praxisnahe Fortbildung gewünscht. Und da kommen Case Reports ins Spiel: Ein konkreter Fall, ein ganz konkretes Vorgehen und dann das Ergebnis - das ist praxisnaher als eine Kaplan-Meier-Kurve. Dazu kommt, dass "case-based learning" viel effektiver ist als abstraktes Lernen. Zusammen mit einem spannend geschriebenen Fall (z.B. „Eine 17-jährige Schülerin erleidet eine Bewusstlosigkeit. Ihre Banknachbarin beschreibt, dass die Pausenglocke geläutet hätte und damit die Mathematikstunde zu Ende ging, als die 17-Jährige plötzlich und völlig unvermittelt im Sitzen zusammensackt und mit Karacho vom Stuhl fällt.") 
bleibt auch eine komplexe medizinische Information im Gedächtnis. Wenn dem Gedächtnis nur die Fakten angeboten werden (also in diesem Beispiel: „Einer rhythmogenen Synkope beim Long-QTSyndrom Typ 2 geht häufig ein akustisches Arousal voraus."), ist die Chance auf einen der vordersten Plätze im Langzeitgedächtnis deutlich geringer als bei Kombination mit dem konkreten Fall. Deshalb erinnere ich mich noch nach vielen Jahren an den Kapitän mit Rauschebart bei Herrn von Olshausen, der mit einer Walkingthrough-Angina in die Notaufnahme gekommen ist, und an den Soldaten mit bevorstehendem Einsatz in Afghanistan, bei dem Herr Heidbüchel eine Delta-Welle gesehen hat.

Case Reports und „case-based learning" stellen extrem effektive Formate in der Fortbildung dar, und ihre Opferung zugunsten des Impact Factors hat daher auch Nachteile. Deshalb freue ich mich besonders, dass uns in dieser Ausgabe von $H+E 18$ exzellente Autoren(-Teams) aus Institutionen mit hoher Expertise in der Rhythmologie 18 lehrreiche Fälle vorstellen. Die Fälle sind alle dem Alltagsgeschäft entnommen und geben wichtige Tipps für die tägliche Praxis. Dabei wurden die Themenbereiche supraventrikuläre Tachykardien, ventrikuläre Tachyarrhythmien und plötzlicher Herztod, Synkope, Device-Therapie und Katheterablation abgedeckt.

Die Beiträge sind nicht offiziell in Form eines Quiz gestaltet, aber verraten die Lösung bzw. Diagnose nicht bereits im Titel oder Abstract, sondern erst gegen Ende der Fallvorstellung, sodass jeder Leser überlegen kann, was er an dieser Stelle gemacht bzw. welche Diagnose er gestellt hätte.

Übrigens für alle diejenigen, die die Lösung nicht schon vor der Fallvorstellung verraten bekommen wollen: Bitte nicht die Schlüsselwörter lesen!

Hiermit möchte ich mich ganz herzlich bei den Autoren für die schönen Fälle aus ihrer Praxis und die tollen Manuskripte bedanken und wünsche den Lesern dieser Ausgabe von $H+E$ viel Spaß und Erkenntnis bei der Lektüre!
$\mathrm{Ihr}$

Achal

Carsten Israel

Korrespondenzadresse

PD Dr. Carsten W. Israel

Klinik für Innere Medizin - Kardiologie, Diabetologie \& Nephrologie, Evangelisches Klinikum Bethel

Burgsteig 13, 33617 Bielefeld, Deutschland

Carsten.Israel@evkb.de

Interessenkonflikt. C.W. Israel gibt an, dass kein Interessenkonflikt besteht. 